Manuscript for a special issue of Current Opinion in Solid State and Materials Science on Mesoscale Science, G. Crabtree and J. Sarrao, ed. (9/4/14, revised 12/17/2014)

\title{
Materials Aging at the Mesoscale: Kinetics of Thermal, Stress, Radiation Activations
}

\author{
Michael P. Short ${ }^{1, *}$ and Sidney Yip ${ }^{1,2}$ \\ ${ }^{1}$ Department of Nuclear Science and Engineering \\ ${ }^{2}$ Department of Materials Science and Engineering \\ Massachusetts Institute of Technology, 77 Massachusetts Ave., Cambridge, MA, 02139, USA
}

The complexity of materials aging may be seen as a result of the interplay between several activation processes operating on multiple spatial and temporal scales. Though the disciplines involved may seem disparate at first, material aging fundamentally could be linked by the same set of underlying activations and responses of the system. We examine how recent studies of shear-induced deformation and rheological flow initiated in the soft-matter community can be leveraged to probe the mechanisms of radiation damage in nuclear materials. Bridging these two traditionally separate areas of research demonstrates the emerging notions of mesoscale science as a research frontier concerned with linking macroscale behavior to microscale processes in driven systems. We suggest the combining of microstructure-sensitive measurements with fundamental theories and mechanism-specific simulations is essential to addressing metastable materials responses of strongly activated states.

Keywords: mesoscale science, self-organized criticality, void swelling, colloidal systems, activation

\section{The Mesoscale Science Frontier}

In the past two years a frontier for materials research at an intermediate regime between microscopic and macroscopic scales has emerged with the prospect of scientific advances coupled with technological impact [1][2][3]. Many types of materials are known for their functional behavior on the macro-scale. In these systems the molecular interactions also have been thoroughly studied. What is missing is a quantitative understanding of the mechanisms underlying the cause-effect relation between molecular interactions and functional behavior. If such connections could be established, it would enable materials behavior at the systems level to be optimized by knowledge-based manipulation at the micro- and mesoscopic levels. The bridging of the micro-macro gap is the essence of the Mesoscale Science (MSS) frontier.

Since the initial recognition of the "the middle way" as a missing conceptual link [4], there have been extensive discussions of the many facets (hallmarks) of MSS, and the different problems

\footnotetext{
*Corresponding Author: hereiam@mit.edu
} 
that illustrate the breadth of this fundamental notion [2]. These efforts notwithstanding, MSS remains an open-ended idea inviting further definition, scrutiny, and demonstration of its merits. It is in this spirit that we offer additional characterization of this scientific frontier and a perspective focused on time-dependent materials deformation.

While a single organizing principle capable of defining MSS seems to be an unlikely prospect, we believe it is nevertheless appropriate to recall the discussions surrounding the formulation of a unifying concept in non-equilibrium statistical mechanics known as self-organized criticality (SOC) [5][6][7]. The hypothesis of SOC was that very different physical systems, ranging from sand dunes to spring networks to even stock markets, are driven by a combination of external forces and internal interactions to special ('critical') states of the system. These states have universal character in that their statistical properties show power-law scaling with the same exponents, the implication being their temporal evolutions are not controlled by any single event. SOC attracted broad interest in the physics community because it appeared to provide a general measure of system complexity involving two concepts, one of self-organization and the other of critical behavior, each with its implications regarding how natural phenomena evolve in time. Since a precise mathematical formulation of SOC in non-equilibrium statistical mechanics is still not available [7], it appears reasonable not to expect anything different in looking for new underlying principles governing MSS. This is in contrast to the principles underlying the free energy or the partition function in thermodynamics, which may be considered the equilibrium counterparts to SOC [7], or perhaps to MSS. On the other hand, SOC and MSS should share their reliance on threshold (metastable) behavior and the dominating influence of fluctuations. In particular insights from SOC remind us of the importance of self-organizing processes in describing the microstructure evolution relevant to MSS. For example, the presence of ratedependent response to a driving force, or memory effects, is a hallmark of MSS. These ratedependent processes are often governed by long-range interactions between defects on two major length scales, one pertaining to the constituent particles of the system (microscale), the other to activated complexes or defect clusters (mesoscale).

We see in MSS an emphasis on microstrucrual defects that result from the combined effects of strong internal molecular interactions and external driving forces. Referring to them as selforganized defects (SOD), we propose the characteristic spatial-temporal scales on which they evolve should define the relevant scales for MSS. The SODs, once formed, will not likely evolve in isolation in any realistic scenario; rather they will self-organize to further multiply or agglomerate, triggering larger-scale structures displaying their own metastable behavior in the presence of an activated environment. Thus, a characteristic behavior of MSS is a system which is evolving in a continuous state of activation. What are examples of such defects, and what is meant by their evolution? While we provide illustrations in the following discussions of yielding in soft matter and radiation damage in nuclear materials, these are also the questions to motivate future research in MSS.

\section{Nonlinear Rheology of Soft Matter}

Colloidal dispersions represent one of the simplest classes of materials for which the interplay between dynamical behavior of dense fluids and externally controlled flow can be investigated. As soft matter they also have widespread applications in all forms, plastics, paints, slurries, etc. 
[8][9]. A basic challenge in nonlinear rheology is to relate the microscopic structure and dynamics to the macroscale stress and flow behavior. Above a certain packing fraction, dense colloidal fluids are expected to display a yield stress and a time delay in hydrodynamic diffusion. Similarly, the onset of flow beyond a critical shear strain is generally attributed to the breaking up of local cages surrounding each particle, and correlated particle rearrangements. Microstructure evolution characteristics like these make the study of nonlinear rheology a research enterprise in which experiments, theoretical modeling, and particle simulations play complementary roles. The advantage of combining experiments with theory and simulation is that one can probe more fully the relation between the macroscale behavior and the underlying mechanisms at the microscale. By this we mean molecular-level details that theory and simulation can provide are useful for interpreting time-dependent macroscale measurements, which by themselves provide insufficient insight to the underlying microstructure evolution. An area in which this awareness is beginning to emerge is the study of chemical structure and mechanics of cement. A molecular model of the binder phase of cement paste is being developed [43] as a basis for interpreting various measurements of structural and physical properties [44][45]. At stake is the link between microscopic simulations and macroscale behavior, and the ability to optimize the design of the binder for durability and environmental impact [46].

Two recent attempts to quantify the micro-macro relation illustrate the benefits of integrating a triad of research capabilities. First, the onset of yielding during start-up shear was investigated by subjecting suspensions of PMMA particles (267 nm and $788 \mathrm{~nm}$ in size) to rheometry and confocal microscopy measurements combined with Brownian particle dynamics simulations [10]. Then the onset of stress relaxation in a flowing suspension when shear rate is abruptly switched off was analyzed by a highly sophisticated theoretical analysis in conjunction with molecular dynamics simulations, in addition to macroscopic rheological measurements [11]. In complementary ways both studies exemplify the essence of microstructure evolution on the mesoscale.

Figure 1 shows the stress-strain variation at several shear rates, expressed in terms of the Péclet number, $\mathrm{Pe} \sim \dot{\gamma} \tau$, where $\dot{\gamma}$ is the shear rate, and $\tau$ is a relaxation time. Each measurement gives a peak (yield) stress $\sigma_{\mathrm{y}}$ and a corresponding strain $\gamma_{\mathrm{y}}$. A higher yield stress is obtained with increasing shear rate, a behavior known as stress overshoot. Note also that the yield strain increases with the shear rate. 


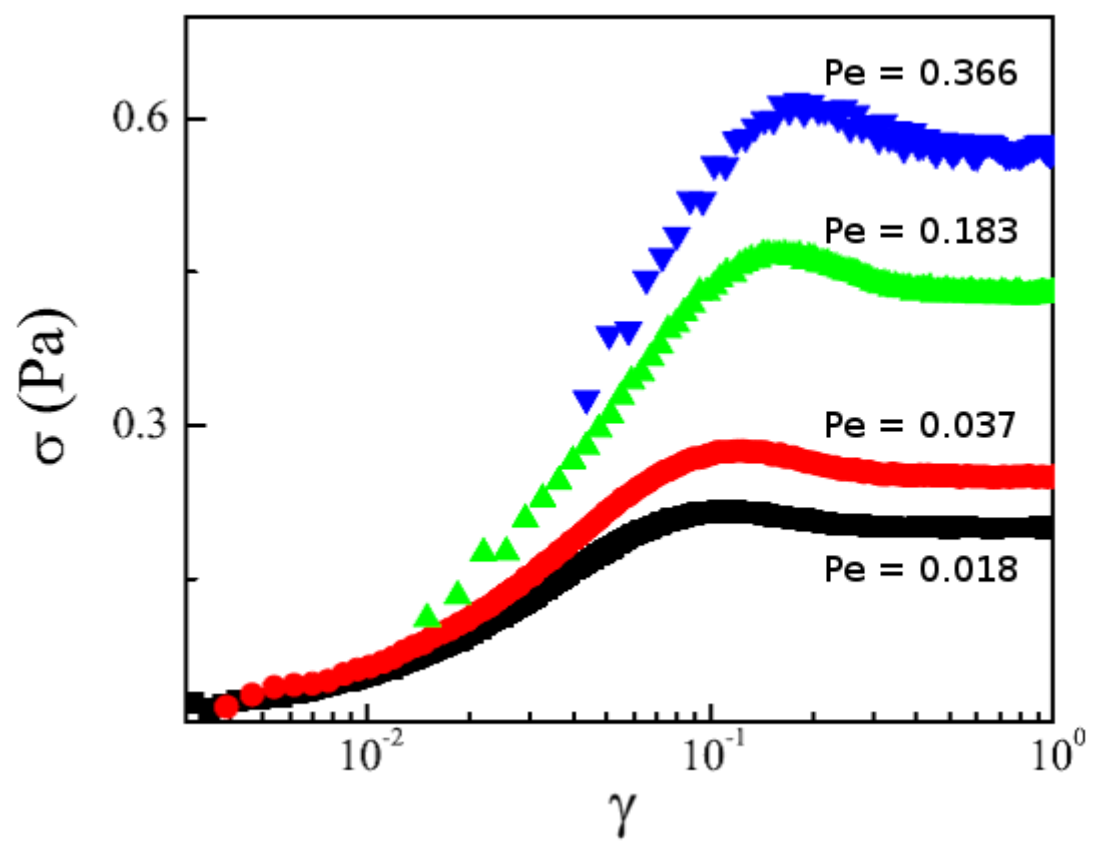

Figure 1: Rheometry data on yielding of a colloidal (PMMA) glass under shear showing stress overshoot and yield-strain shift with shear rate (expressed in Péclet number) [10]

A qualitative interpretation of the yielding behavior shown in Figure 1 suggests a certain amount of time is needed for the colloidal particles to align themselves in response to the imposed shear. This would be an indication of memory effects. Intuitively one expects that if the shear rate is too fast for the process to complete, then the system will act more rigidly and with a time delay. This can be regarded as a general consequence of rate or 'memory' effects associated with microstructure evolution. The system response should also depend on the amount of space locally available to the particles, or at the macroscale on the free volume (packing fraction) in the system. This simply means microstructure evolution has corresponding manifestations in the spatial and temporal degrees of freedom. An example of the equivalence can be seen in Figure 2, in the form of the flow curves at two packing fractions (2(a)), and the yield stress variation with the packing fraction, Fig. 2(b) [12]. If one were to ask about the underlying mechanisms of yielding according to a theoretical description, or about direct particle simulation on the same scales as the measurements, then the results for such discussions have become available only recently. 

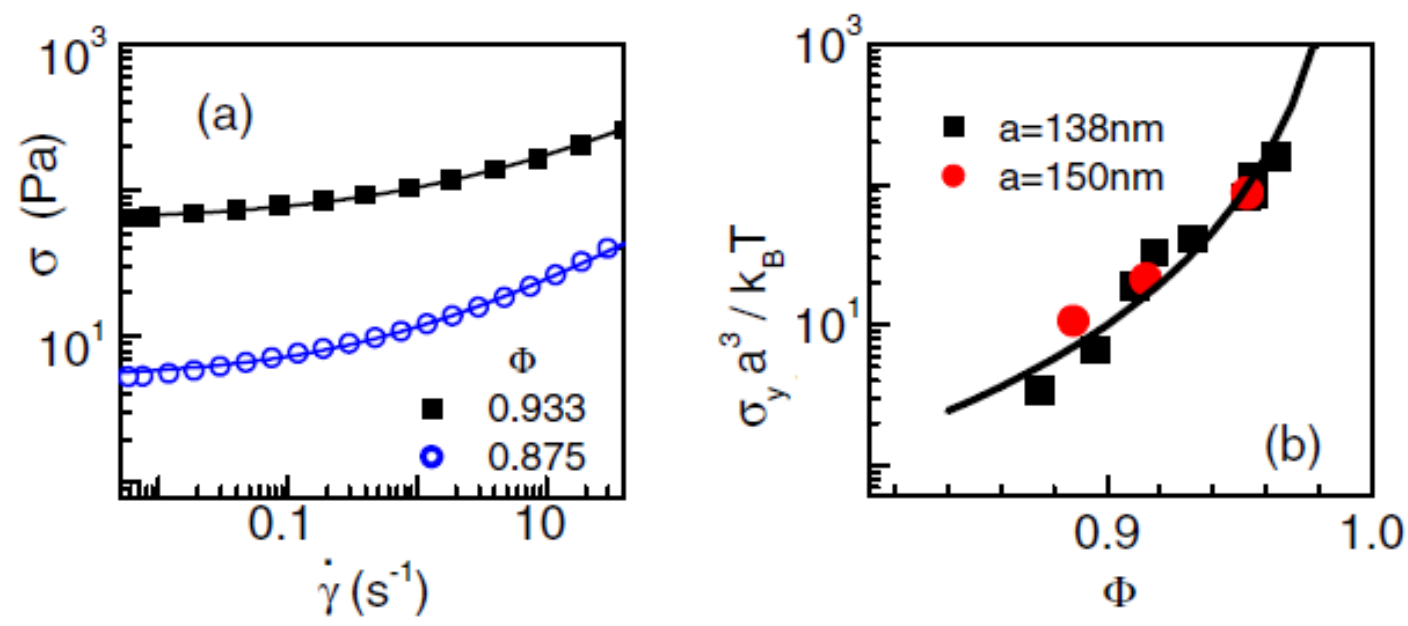

Figure 2: (a) Experimental flow curves of colloidal glass showing yield stress increase with strain rate at two volume fractions, (b) normalized yield stress increase with volume fraction for two colloidal particle sizes [12]. Curves are fits through the data (a), and a scaling expression (1$\Phi)^{-3}(\mathrm{~b})$.

In viscous liquids or dense suspensions a typical mode of atomic motion is the rattling of a particle temporarily confined in a cage formed by its near neighbors [13]. These vibrations are transient since the cages form and breakup spontaneously, their lifetimes being determined by the local configurational environment, the interparticle interactions, and the forces of thermal and stress activations. The presence of the caging effect is directly reflected in the time variation of the velocity autocorrelation or the mean-squared displacement function, which are quantities that can be extracted from scattering experiments or computed from molecular simulations [14].

Figure 3 shows the mean squared displacement obtained in a molecular dynamics simulation of water molecules in a porous silica gel, at density $0.5 \mathrm{~g} / \mathrm{cc}$ and a temperature of $100 \mathrm{~K}$ [14]. The initial low values of MSD indicate the molecules (on average) are trapped in a cage with a lifetime of $\sim 50 \mathrm{ps}$. after which they undergo diffusive displacements (MSD linear in tine) until a second cage is formed approximately 50 ps later. A Voronoi tessellation of the particle coordinates, shown in the inset, provides confirming evidence that the different neighbor configurations are involved in the two trapping events. At lower temperatures the caging effect is found to persist over longer periods as one expects for a thermally activated process. 


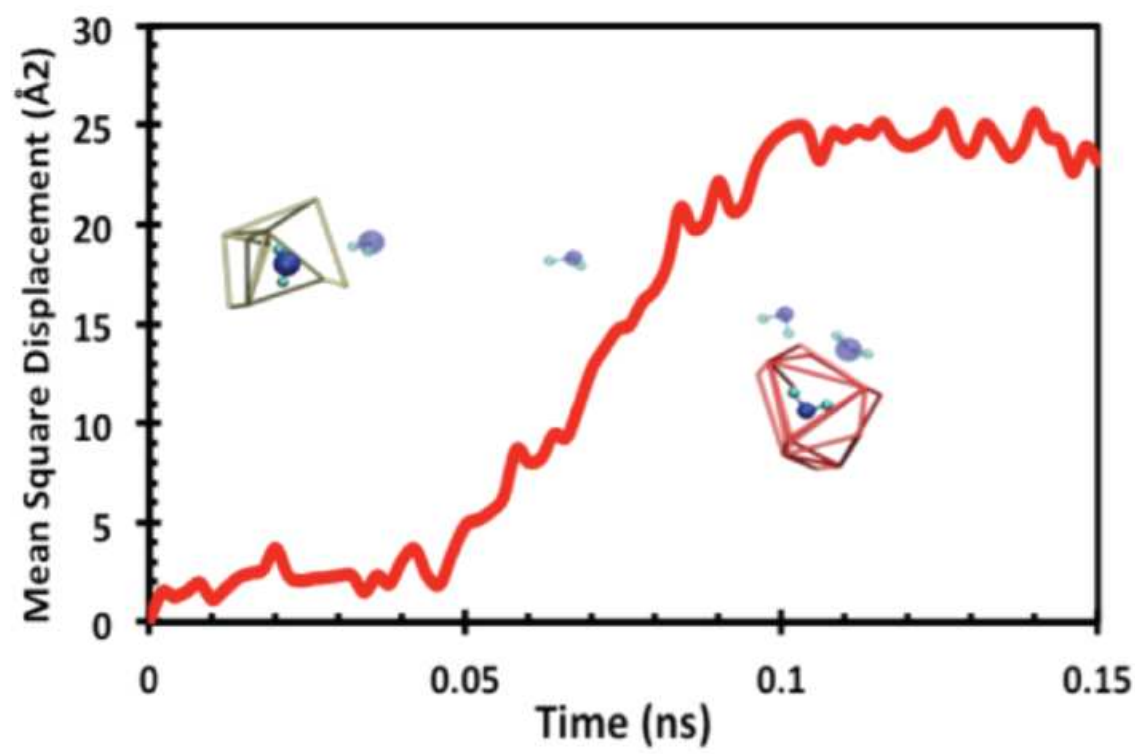

Figure 3: Mean square displacement of water molecules in a silica gel showing trapping effects for a period of about $50 \mathrm{ps}$, followed by diffusive motion for about $50 \mathrm{ps}$ and then a second trapping [14].

Turning to the results where the applied shear on a colloidal suspension in steady-state shear flow is suddenly removed [11], we follow the time response of the ensuing stress relaxation as described by the self-consistent mode coupling theory [15] extended for nonlinear response [16]. Figure 4 shows that the normalized stress level ( $\mathrm{R}$ is size of colloidal particle) does not respond instantaneously upon the cessation of shearing. The onset of relaxation is first of all very sensitive to the microstructure state of the system. The three volume fractions chosen for analysis are quite close to the values across the ideal glass transition $(\Phi \sim 0.52)$. According to the extended mode-coupling theory, at $\Phi=0.52$ the system is structurally arrested (jammed) to the extent that the stress is unable to relax in the time interval indicated. On the other hand, at lower volume fractions of $\Phi=0.51$ and 0.515 , the onset of stress relaxation becomes visible in the same interval, and the decay begins earlier for lower volume fractions. This behavior is another manifestation of memory effects, indicating time is needed to organize the internal relaxation, and the persistence time depends on both the particle packing and the shearing deformation. With regard to the strain rate effect, the residual stress level is seen to increase with increasing strain rate which is consistent with the behavior previously discussed regarding Fig. 1.

Fig. 4 also illustrates an essential aspect of MSS in the explicit focus on understanding the temporal behavior of a system property (the stress). By directly calculating, measuring, or simulating this response at the appropriate spatial-temporal scales, one can gain access to valuable insights into the underlying mechanisms at the lower scales. Thus the greater persistence of the residual stress at the higher shear rate would indicate stronger cages. Conversely, it would follow that external shear is disruptive to maintaining the local cages. 


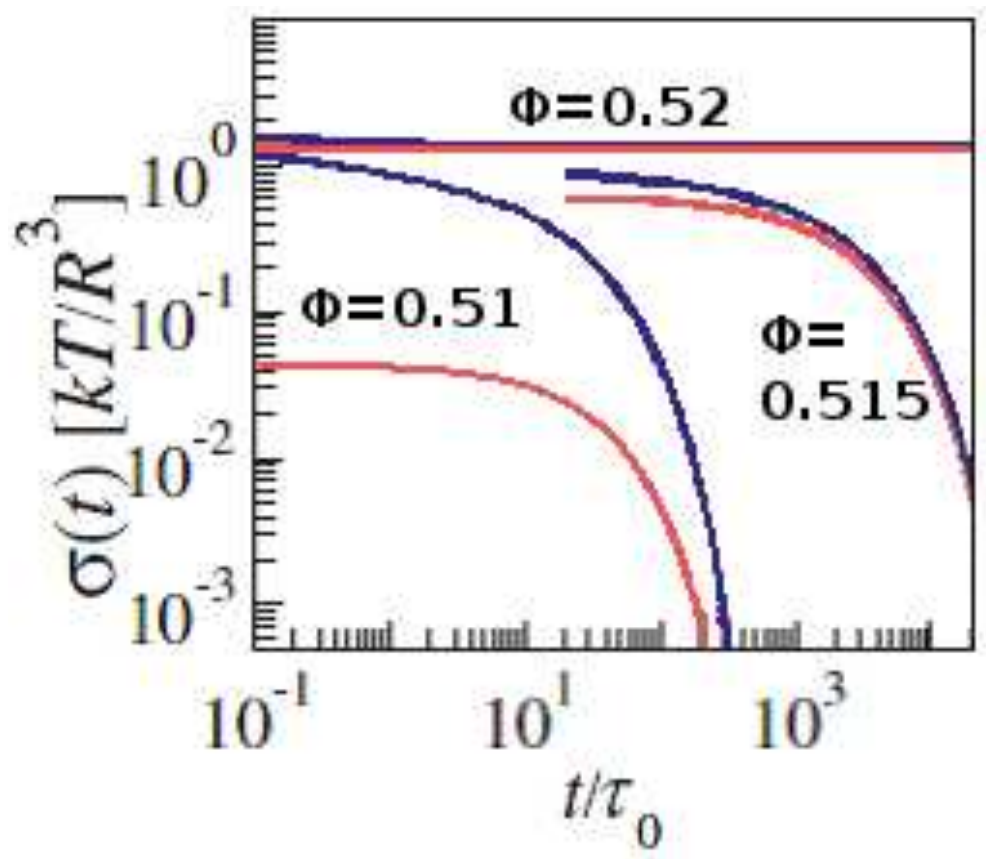

Figure 4: Onset of relaxation of normalized residual stress in a colloidal suspension in steadystate shear flow upon the sudden removal $(a t \mathrm{t}=0)$ of the applied shear, with time normalized by a relaxation time $\tau_{\mathrm{o}}[11]$. Curves are for three volume fractions discussed in the text, the largest being a glassy state and the other two are liquid states described theoretically by an extension of the mode-coupling formalism [11]. Also shown is the dependence on shear rate, $1 \mathrm{~s}^{-1}$ (blue) and $10^{-4} \mathrm{~s}^{-1}$ (red).

The fact that residual stresses now can be theoretically analyzed is itself a significant advance. Additionally, molecular dynamics simulations and experimental results have been compared to the theoretical predictions, thus demonstrating the complementarity of the three techniques [11]. Figure 5 shows the time evolution of the mean squared displacement function for the nonequilibrium scenario (steady state shear flow) and two equilibrium systems, one glassy and the other a dense liquid. In the liquid, a distinct plateau indicates local trapping of the particle before the onset of continuous diffusion. For the glassy system where structural relaxation is considerably delayed, the transition to diffusion is expected at much later times than the indicated range in the figure. Compared to these results, we see only a hint of the caging effect in the flow state (NESS). This is evidence that particle mobility is sensitive to external perturbations, the kind of insight that would be difficult to extract from experimental data without corroborative details from modeling and simulation (see also [10]). 


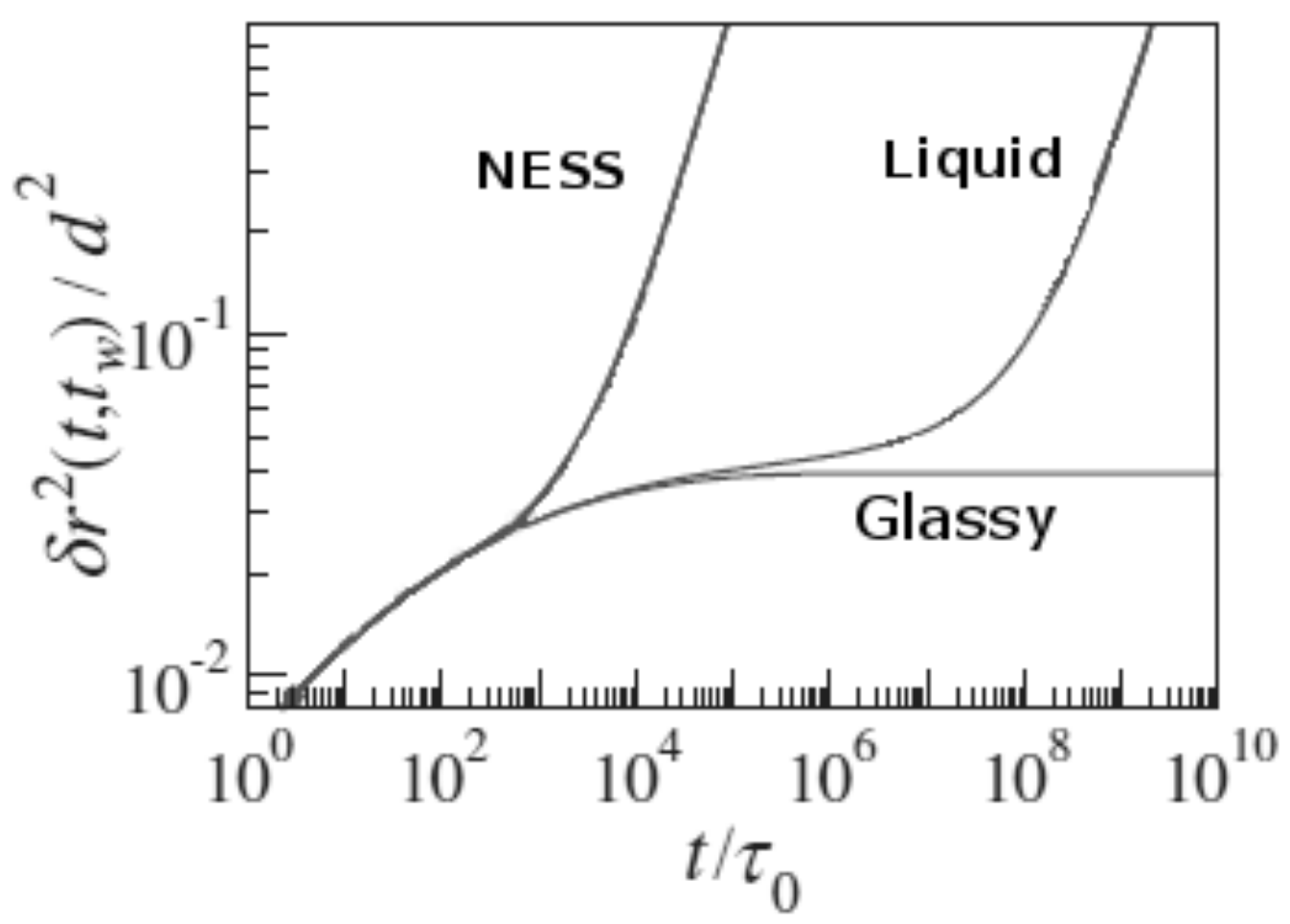

Figure 5: Mean squared displacement of colloidal particles in a dense suspension showing different time evolutions. The NESS curve is for the non-equilibrium system in steady-state shear flow, while the other two refer to equilibrium liquid and glassy (structurally arrested) states. Time is normalized by a relaxation time and does not include waiting time to eliminate transient effects.

The interest in interpreting shear-rate effects in colloidal flow, or memory effects more broadly, is an example of the MSS emphasis on time-dependent behavior of the system reflecting an underlying transition in its microstructure, again as a consequence of self-organization. In this case it is the interplay between the aggregation of the colloidal particles and local environmental forces disrupting the steady-state. We now turn to a distinctly different area of study from soft matter, but we still ask the same question, "How can one understand and possibly predict a significant change in the system response?"

\section{Radiation Damage in Nuclear Materials}

Swelling and creep under irradiation may be considered to be broadly analogous to yielding under shear in that both are rate-dependent microstructure responses to external perturbations. The responses result in damage accumulations, which in turn trigger complex macroscale behaviors. Dimensional distortions of structural nuclear materials affect the safety of existing nuclear systems as well as the future reliability of nuclear power. Because of its direct industrial relevance for over five decades, a great deal is known about radiation damage phenomena in metals, particularly alloys with FCC and BCC structures [23]. Recent reviews of the theory of radiation damage [23][59] along with extensive data and interpretation [23] indicate this provides an opportunity for research into the mechanisms of time-dependent microstructure evolution in 
the presence of irradiation effects. Even though microscale [18][24][59] and macroscale [36] descriptions of void swelling exist, it is still not feasible to predict a priori the sudden onset of void swelling as functions of imposed state variables.

Consideration of void swelling begins with the basic damage due to atomic displacements produced by the ballistic interaction of radiation with structural materials. As depicted in Fig. 6, the microscale processes are the production of point defects, vacancies and interstitials, while the macroscale behavior is that of volumetric swelling. From the appearance of a thermal spike as a single radiation-damage event (femotseconds) to the time for vacancies to combine and form voids (nanoseconds), to the time for voids to migrate and accumulate (seconds to years) [17], the understanding of the individual processes and how they are connected to each other is a formidable challenge. The processes in (a-e) are well-understood and can be linked using multiple simulations, though the definitive link from (e) to (f) remains difficult to predict in real materials.

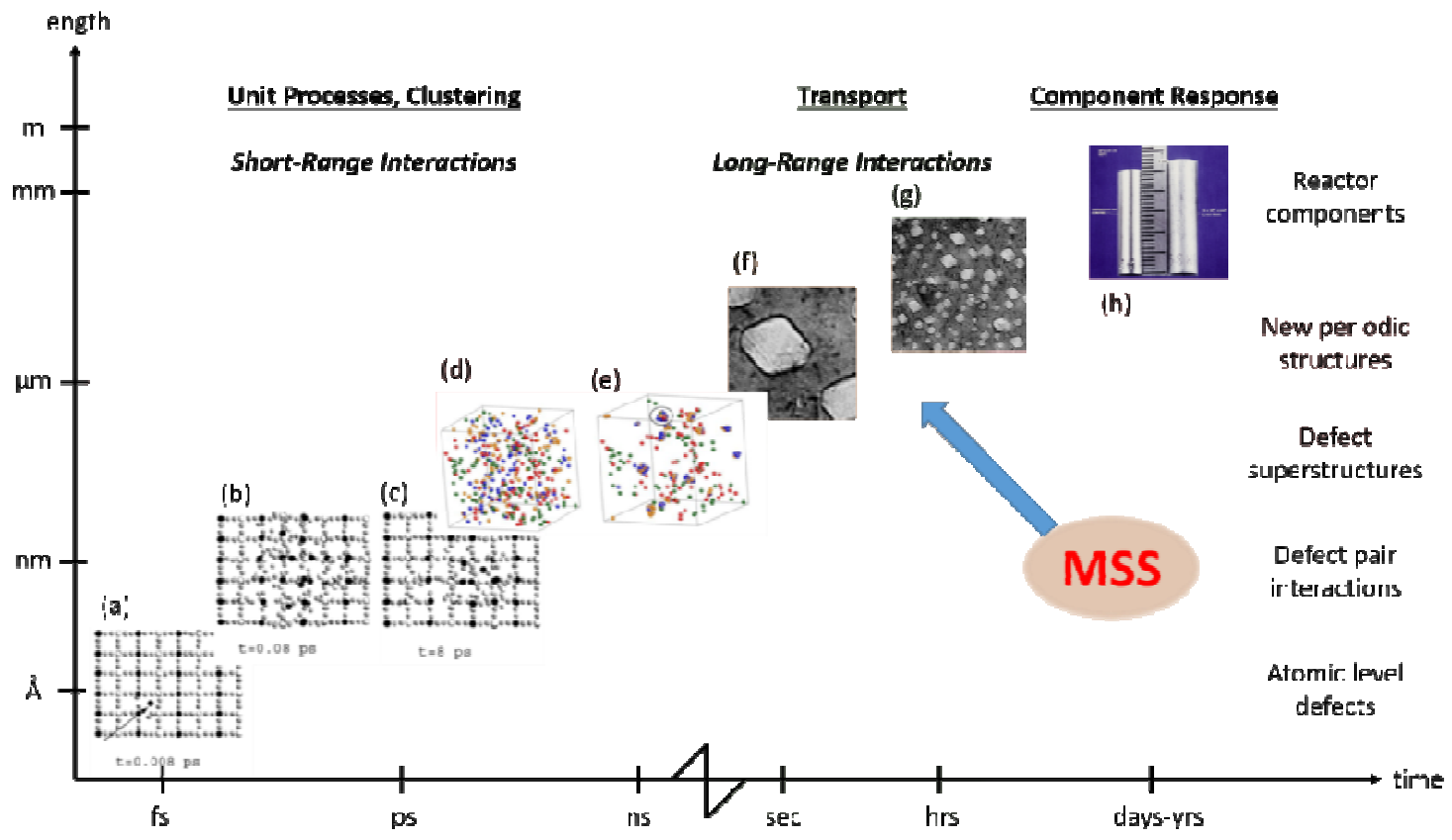

Figure 6: Spatial-temporal scales of processes involved in void swelling. Radiation interaction produces primary knock-on atoms (a) which give rise to a thermal spike (b) followed by subsequent annealing (c). These processes occur quickly to form lattice point defects (d) which relax under short-range interactions into local defect structures (e). The latter then act as nucleation sites for voids (f), which further self-organize into a semi-regular lattice (g) through long-range interactions. As the voids grow and the lattice spacing evolves, one finds macroscopic swelling in the material component (h). References: [19] for (a-c), [20] for (d-e), [37] for (f-h).

Three distinct spatial-temporal scales are at play leading to macroscale void swelling over the lifetime of a nuclear reactor (30-80 years). The production of point defects - vacancies and interstitials - is the unit process common to all forms of radiation damage. The initial radiationmaterials interaction produces a primary knock-on atom, which then in a sequence of following 
collisions knocks several atoms out of their lattice sites. However, after periods of nanoseconds many displaced atoms find vacant lattice sites to occupy or recombine with other defects \& sinks, so the number of vacancies and interstitials that remain after a few picoseconds is rather small compared to the initial displacements. As the remaining vacancies agglomerate, they form clusters and eventually voids.

Void swelling originates in the difference between the agglomeration and interaction of interstitials of one or two dimensions and of vacancies, mostly in three dimensions such as cavities. This distinction gives rise to the accumulation of void space, accompanied by a density decrease or volume increase. Cavities in the form of 'voids' are essentially vacuum- or gas-filled holes. Cavities which contain hydrogen or helium are often characterized as bubbles. Voids in the microstructure can interact directly, via thermal emission of single vacancies, or through a dislocation field. These may be taken to be the relevant self-organized defects for MSS studies, with spatial-temporal scales dependent on the state variables specifying the irradiated system: equilibrium vacancy concentration, temperature, material composition, degree of cold work, etc.

This multiscale materials evolution process is quite analogous to the description of colloidal flow under shear in Section 2, whereby the constituent system particles (colloids) form metastable, transient superstructures (local cages), which dictate the macroscale response of the system. In void swelling, the vacancies form small, metastable vacancy clusters, which will under some conditions decay should the driving force (continuing irradiation) be switched off, or evolve into more stable superstructures (voids) which then cause the material to swell on the macroscale.

The theory of radiation damage in matter has a long history of development based on rateequation formulations with various approximations for sources and sinks; essentially it is also a form of transition-state theory with detailed account of the point defect concentrations [24]. Recently the role of self-interstitial atom clusters and their one-dimensional mobility, as well as a production bias of isolated vacancies vs. interstitials and their clusters, has been clarified in the problem of irradiation defect growth. Simulations show the metastability of small-vacancy clusters, up to about 52 vacancies, whereas ion beam irradiation experiments give very different results [23][24]. The role of point-defect cluster evolution in space and time varying local environments again examplifies the emphasis on self-organized defects in MSS.

The dynamic response of present interest is the variation of void swelling with radiation damage shown in Figure 7. The standard unit of radiation damage is the dose or displacement per atom $(d p a)$. It is a calculated quantity, whereby a value of one specifies the flux of incoming radiation needed to knock out (by radiation-atom interaction) every atom from its lattice site once on the average. The fact that $d p a$ is not an experimentally accessible quantity makes the interpretation of any irradiation results expressed in this way somewhat ambiguous. In Fig. 7(a) we see during the early stages of irradiation, swelling can be detected at the $1 \%$ level. As the damage accumulates, swelling can abruptly increase. Note that a dose of $\sim 150 \mathrm{dpa}$ is in the range of radiation dose that a fast reactor component would experience over the reactor lifetime. Thus swelling can reach tens of percent near the end of the service period. This sudden upturn in swelling has been seen in various conditions of alloy composition, crystal structure, irradiation temperature, amount of cold work, etc. [37]. Figure 7(b) shows the sensitivity of the responses to irradiation temperature for the same specimens. While the amount of swelling may differ by a 
factor of two, the behavior of an abrupt increase is a well-established phenomenon. Its explanation and prediction in terms of microstructure evolution processes at the mesoscale constitute a challenge for the MSS community.
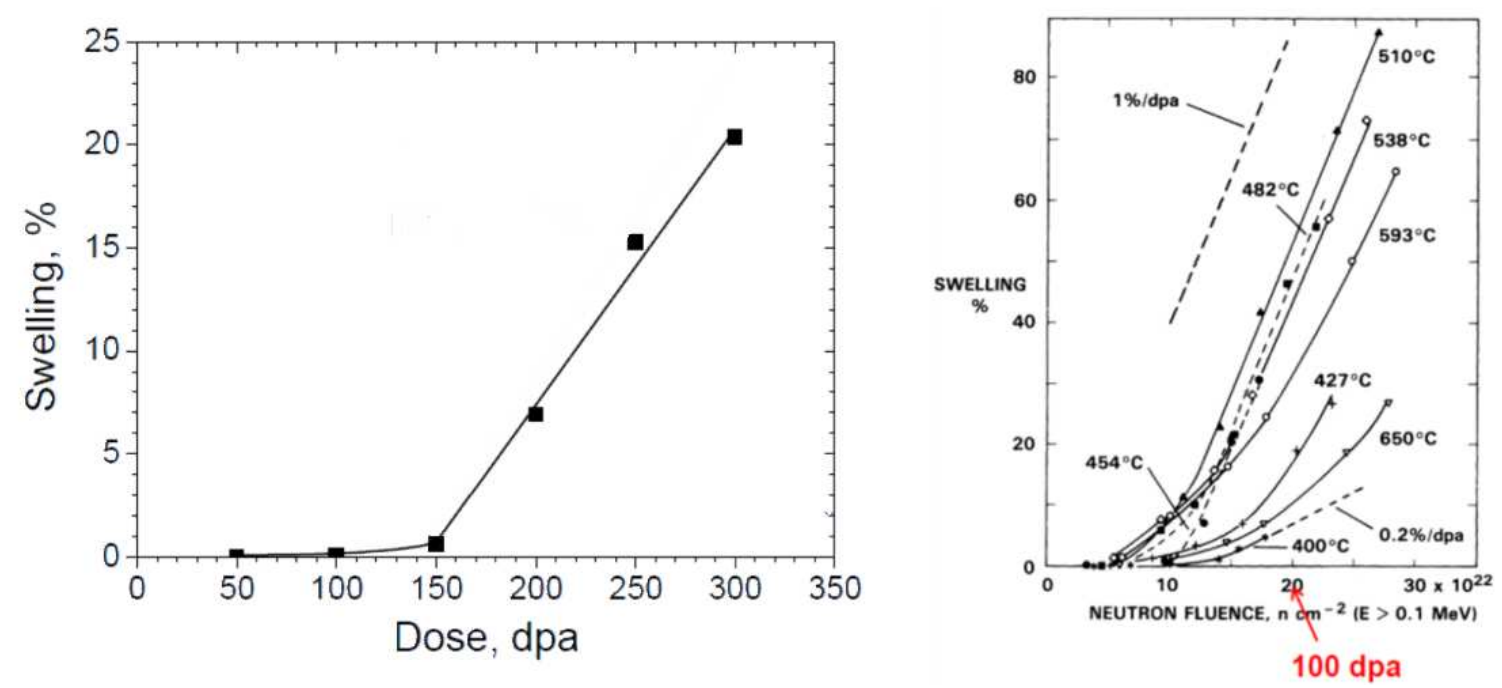

Figure 7: Dose-response curves showing the sudden upturn in void swelling with applied DPA [37]. In (a), the "knee" in the curve clearly indicates a change in the underlying mechanism governing the macroscale behavior. In (b), swelling curves for the same material at different temperatures exhibit very different times to the onset of faster swelling.

The threshold or transitional behavior depicted in Fig. 7(a) may be viewed as a form of critical (abrupt) behavior for which the descriptive term of "avalanche" comes to mind. This term has appeared in the current literature regarding materials yielding and deformation, although not necessarily always consistently [58]. We have referred to this behavior as an "upturn." Other terms that have been used, each with its own context but all having in mind the same phenomenology, are "crossover" [60] and "breakaway" [61]. The problem context in which such behavior has received considerable attention include the temperature variation of the shear viscosity of supercooled liquids [27], the yield stress upturn at high strain rate in shock impact [28], and the stress variation of strain rate in thermal creep [29]. Based on our earlier reference to SOC, it is tempting to view the two kinds of deformation phenomena in this perspective, the yielding response under shear and swelling response to radiation damage, as belonging to a broad class of MSS phenomena involving microstructure evolution of activated states of matter.

The critical dose where the swelling upturn occurs shows considerable variations with the state variables specifying the dose rate, alloy composition, irradiation temperature, and the amount of cold work, some of which can be seen in Figure 8 . This sensitivity suggests the magnitude of swelling is an integral response which is likely to be influenced by more than a single mechanism. It is known that pure metals undergo swelling at significantly lower doses, while their behavior at high doses remains to be studied [37]. Figure 8(b) shows a dose-rate effect for which the interpretation is under discussion [38]. A slow swelling rate at a higher dose rate suggests the slower response is due to faster activation which leaves less time to self-organize. A similar type of explanation of temporal localization of external perturbation has been used to 
interpret shock impact data where the yield stress in a metal increases abruptly when shear rate reaches a characteristic value [54].
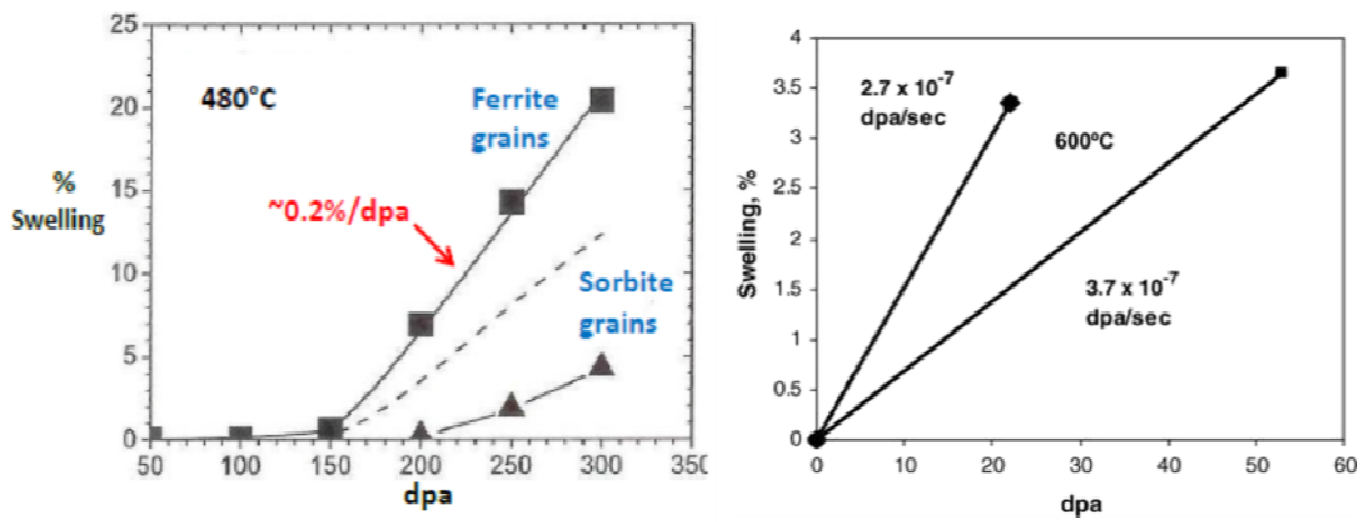

Figure 8: Curves showing the significant change in swelling response due to long-range interactions. In (a), different crystal structures experience an upturn in swelling at very different doses [37]. In (b), changing the dose rate causes different amounts of swelling for the same applied dose [38], suggeting that interactions between neighboring thermal cascades result in reduced formation of mesoscale voids.

While neutrons are the radiation of concern in materials damage in nuclear reactors, ions ranging from light (protons) to heavy (up to uranium) are used to achieve accelerated damage rates in materials testing. However, this introduces potential issues of sample overheating and variations in the penetration depth. As a result one encounters an imbalance in spatial distributions of defects which makes the interpretation of experimental swelling profiles dependent on modeling and simulation. Here one sees also the important effects of interactions between the agents of microstructural evolution and external variables (injected ions, free surfaces) aid in explaining complex swelling data. A particular scenario is depicted in Figure 9. Fig. 9(a) is a TEM micrograph of irradiated specimens of pure iron showing a distribution of voids. At $105 \mathrm{dpa}$ the peak dose is initially estimated to be located about 1 micron into the irradiated surface (free surface is located on the right), considering only vacancy production in a Monte Carlo simulations. The vacancies appear to pile up to form voids of certain sizes with a rather tight characteristic spacing. The depth profiles seen in Fig. 9(b) consist of measurements at three irradiation doses along with two simulated distributions, one for the incident Fe ions and the other for the displacement of the lattice Fe atoms in the thermal spikes. It is apparent the measured swelling profiles have peaks closer to the surface of the irradiated specimens than the simulated distributions. Moreover, as the doses increase (at 70 and $105 \mathrm{dpa}$ ) a splitting in the swelling profiles can be resolved, a feature that is not explained by atomic scale considerations alone.

Interpretation of the data is facilitated by direct simulation of ion transport and defect migration by the finite element method, and considering the problem from an MSS point of view. In a 
recent investigation long-range interactions in defect evolution have been shown to govern the distribution of injected interstitials, and the suppression of sub-surface swelling [33].

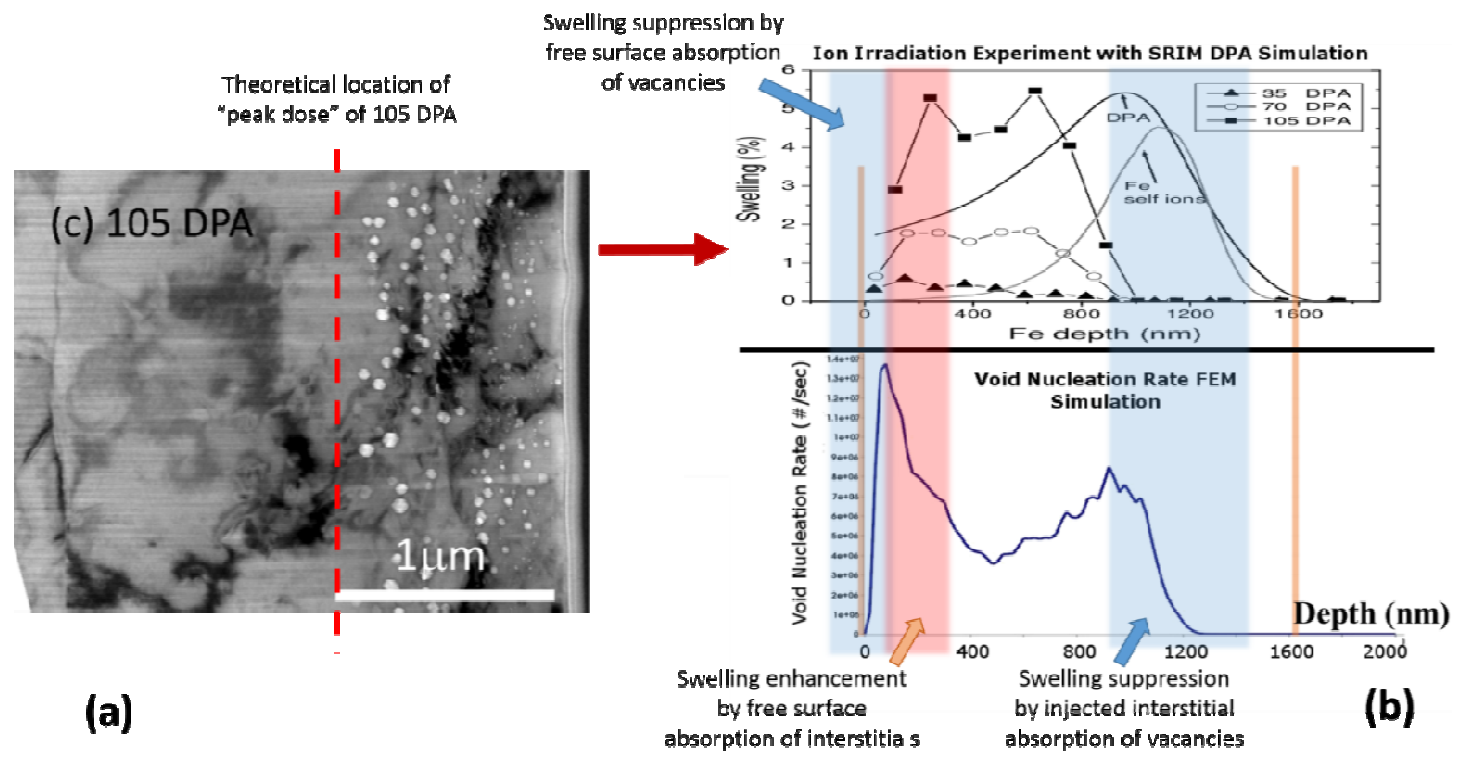

Figure 9: Response of pure iron to self-ion irradiation at different doses, making long-range, mesoscale interactions evident by the swelling curve being different from the radiation damage curve imposed by the incoming self-ions [33]. In the top half of (b), the smooth curves denote atomistically simulated damage rates (DPA) and injected interstitial concentration (Fe self ions), respectively, while the symbols represent experimental measurements of void swelling at different peak doses. The bottom half of (b) shows a finite element simulation of the spatially dependent void nucleation rate, which aligns well with the experimental data [34].

\section{Returning to}

Figure 9(b), at depths of 0-100nm, the free surface is effective in removing vacancies as well as interstitials, leaving a void-denuded zone similar to that found near grain boundaries and dislocation walls [35]. Between 100-400nm the surface can continue to attract the more mobile interstitials, but not the vacancies, thus leaving fewer interstitials for recombination. Between 1000-1600nm, the injected interstitials (the self-ions coming to rest in the material) create a defect imbalance by annihilating vacancies and thereby suppressing void swelling. These processes therefore give rise to a spatially distributed range of swelling suppression and enhancements as indicated. Recent finite element predictions which consider the spatial void nucleation rate as functions of the skewed production and migration of point defects, including the injected interstitials, predict the split swelling peaks very well. In this way one can understand the appearance of the double peak in swelling profile at high $d p a$. 


\section{Mesoscale Science in Materials Aging}

MSS provides a platform to address materials aging problems where radiation effects couple with thermal and stress activations to collectively drive the microstructure evolution at the mesoscale. MSS is similar to SOC in that both subscribe to a broad outlook on understanding how physical processes evolve in fundamental ways that are common in systems traditionally considered to be quite different. By focusing on threshold behavior at the macroscale, one can formulate a set of challenges to further stimulate innovations in developing our research capabilities at lower length scales. It seems clear that new experimental techniques should be complemented by multiphysics modeling, coordinated with multiscale simulations, and ultimately integrated into a self-consistent theory. Theory in particular can integrate our knowledge and insight in powerful physical principles and models, such as treating thermal activation though a transition-state theory and master equation formulation that one cannot achieve with only experiments or simulations [62], or formulating an effective temperature model based on thermodynamic principles [63]. On the other hand, simulations can provide details on response to extreme conditions that are difficult or not feasible to observe through actual measurements. The complexity of the self-organized defects and their interactions which we have discussed indicates that synergistic implementation of all three research methods is needed.

As an example, one recently suggested approach to interrogate and experimentally distinguish mechanisms of the upturn in material radiation response is to study the time-dependent surface acoustic waves generated by short laser pulses [35]. This technique, which operates at the length scale of microns and the time scale of seconds, seems well suited to simultaneously probing the elastic and thermal properties of irradiated metals, just microns below the free surface [36]. Considering that existing ex-situ datasets on void swelling are relatively sparse, time-resolved data in situ clearly would be very useful. One may expect that combining this approach with massively parallel molecular dynamics simulations could help in the validation of competing theoretical mechanisms proposed for the onset of void swelling [21]. The ability to acquire insitu laser-induced surface acoustic wave data during irradiation will also greatly increase the speed and throughput of data acquisition, allowing experimental campaigns to cover broader ranges of data with finer resolution, and to resolve coupled effects. Ultimately the integration of mesoscale experiments and simulations seems essential if a theory is to be developed that is capable of predicting the onset of void swelling for new alloys as functions of the starting microstructure and environmental state variables. This would indeed constitute a revolutionary leap in nuclear materials science, where innovation could proceed at speeds closer to those in the semiconductor industry. Unfortunately at present the alternative of multi-year, costly irradiation campaigns is the only viable option [42].

An illustration of the microstructure evolution that one would like to understand in more detail is Figure 4. Explicit time behavior of a system-level response is of fundamental interest, especially when a dramatic contrast (in the stress relaxation in glassy versus liquid systems) can be modeled by theory. Curves of $\sigma(\mathrm{t})$ obtained by experiment, theory, and simulation ideally should be discussed on the same time scale. This is not yet quite feasible at present, because normalizations using different relaxation time constants appropriate to each technique are still necessary. Nevertheless, the attempt to directly compare all three types of results is highly 
commendable, and should be encouraged in future work. The theoretical advances implied by the results shown in Fig. 4 are noteworthy. To our knowledge, self-consistent mode coupling theory is the only formalism capable of describing an idealized dynamical transition between glassy and liquid states [15]. The results of Fig. 4 are obtained from an extension of the original modecoupling theory to non-equilibrium systems [16], the former being a mean-field description in the framework of linear response theory. The key step of the extension is to introduce local fluctuations through a "dynamical correlator" [16] , in a way that justifies calling the new mode coupling approach Integration Through Transients [16]. Other recent theoretical developments in condensed matter physics also point to a similar conclusion, namely, mean-field models are not sufficient to fully describe the role of self-organized defects without introducing some form of tuning mechanism [58]. The implication of these theoretical advances for nuclear materials studies is that new broadly based models that can connect with both experiments and simulations would be very timely.

Similarly, MSS could stimulate the implementation of advanced molecular simulations. The natural disparity in the time scale on which special effects experiments, theoretical modeling, and particle simulations can each readily access presents a major problem in bringing all three techniques to bear on the same microstructure evolution process. This difficulty is well recognized in the broad materials research community. It is particularly serious for particle simulations, where traditional molecular dynamics method are known to be restricted to microscopic times, of the order of nanoseconds. An opportunity exists for acceleration methods based on transition-state-theory [47] and methods of kinetic Monte Carlo ideas [48], and also for metadynamics sampling of the energy landscape through activation-relaxation algorithms is [50][56][57][51].

For a final outlook on materials aging problems, we believe studies of materials degradation phenomena such as corrosion [3][49] and creep [51] could benefit from the perspective of MSS. The science of cement is an interdisciplinary example where societal and environmental impacts can be very substantial. Besides its preeminent role mentioned earlier, concrete is also considered an infrastructure material important for lifetime extension of nuclear power plants. Understanding the molecular basis of the phenomenon of cement setting, the time evolution of the calcium-silicate-hydrate phase of cement paste [52][53] and delivering of a modelingsimulation capability to predict both the deleterious effects of chemical attacks and the beneficial effects of additives (on the set time of hydrated cement paste) would contribute considerably to transforming this unique field of science and technology [44][45]. Capabilities enabled by the targeted combination of mesoscale theory, modeling \& simulation, and experiments will find excellent opportunities in longstanding problems throughout nuclear materials. MSS could be the key to unraveling their myriad of relevant mechanisms, and finding ways to mitigate their deleterious consequences. 
Acknowledgment. SY acknowledges support by the Project on Sustainability of Kuwait's Built Environment of the MIT Center for Natural Resources and Environment, and by Basic Energy Sciences, U.S. Department of Energy award DE-SC0002633, as well as associations with the Concrete Sustainability Hub at MIT. MS and SY acknowledge association with the Consortium for the Simulation of Advanced Light Water Reactors, an Energy Innovation Hub of the U.S. Department of Energy. MS also acknowledges useful conversations with F. Garner of RCI, Inc., L. Shao of Texas A\&M, and G. Was of U. Michigan. SY also acknowledges the hospitality of the Kavli Institute of Theoretical Physics at the UCSB for a program participation.

\section{References}

[1] "From Quanta to the Continuum ..." DOE-BES Mesoscale Science Report, September 2012.

[2] G. Crabtree and J. L. Sarrao, MRS Bulletin (37):1079 (2012).

[3] S. Yip and M. P. Short, Nature Mater, 12:774 (2013).

[4] R. B. Laughlin, D. Pines, J. Schmalian, B. P. Stojkovic, PNAS (97):32 (2001).

[5] P. Bak, C. Tang, K. Weisenfeld, Phys. Rev. Lett. 59:381 (1987).

[6] P. Bak, How Nature Works (Springer-Verlag, New York, 1996).

[7] H. J. Jensen, Self-Organized Criticality (Cambridge Univ. Press, Cambridge, 1998).

[8] R. G. Larson, The Structure and Rheology of Complex Fluids (Oxford Univ. Press, New York, 1999).

[9] J. Mewis and N. J. Wagner, Colloidal Suspension Rheology (Cambridge Univ. Press, Cambridge, 2013).

[10] N. Koumakis et al., Phys. Rev. Lett. 108:098303 (2012).

[11] R. Ballauff et al. Phys. Rev. Lett. 110:215701 (2013).

[12] R. Besseling et al., Phys. Rev. Lett. 105:268301 (2010).

[13] E. R. Weeks, D. A. Weitz. Chem. Phys. 284:361-367 (2002).

[14] A. Shekhar et al., Appl. Phys. Lett. (2014) Supplemental Information.

[15] W. Goetze, Complex Dynamics of Glass-Forming Liquids (Oxford Univ. Press, Oxford, 2009).

[16] M. Fuchs, M. E. Cates, Phys. Rev. Lett. 89:248304 (2002)

[17] G. S. Was. "Fundamentals of Radiation Materials Science: Metals and Alloys." Springer, ISBN \#978-3540494713 (2007).

[18] Y. Fan et al., Phys. Rev. Lett. 106:125501 (2011).

[19] K. O. Trachenko, M. T. Dove. E. K. H. Salje. J. Phys. Condens. Matter, 13:1947 (2001).

[20] D. S. Aidhy et al., Scripta Mater., 60(8):691 (2009).

[21] M. R. Gilbert et al., J. Phys.: Condens. Matter 20:345214 (2008).

[22] S. I. Golubov, B. N. Singh, H. Trinkaus, J. Nucl. Mater., 276(1-3):78-89 (2000).

[23] T. R. Allen, "High Dose Radiation Effects in Steels," in K. E. Sickafus, E. A. Kotomin, B. P. Uberuaga, "Radiation Effects in Solids," Springer, Ch. 5 (2007).

[24] S. I. Golubov, A. V. Barashev, R. E. Stoller, "1.13 - Radiation Damage Theory,” In Comprehensive Nuclear Materials, ed. R. J. M. Konings, Elsevier, Oxford, pp. 357-391, ISBN 9780080560335 (2012).

[25] E. Beckett et al. "Role of Beam Rastering on Microstructural Evolution in Ion Irradiated HT9 Steel." In TMS 2014 Annual Meeting \& Exhibition, Orlando, FL, USA (3/2014).

[26] L. Shao et al. "Impact of Beam Rastering on Void Swelling in Pure Iron." In TMS 2014 Annual Meeting \& Exhibition, Orlando, FL, USA (3/2014). 
[27] C. A. Angell, J. Phys. Chem. Solids 49:863 (1988).

[28] R. W. Armstrong, W. Arnold, F. J. Zerilli, J. Appl. Phys. 105, 023511 (2009).

[29] R. L. Klueh, Int. Mat. Rev. 50:287 (2005).

[30] R. E. Stoller, G. R. Odette. "A composite model of microstructural evolution in austenitic stainless steel under fast neutron irradiation." In Radiation-Induced Changes in

Microstructure, $13^{\text {th }}$ International Symposium, ASTM STP 955 (F. A. Garner, N. H. Packan, A. S. Kumar Eds.), pp. 371-392 (1987).

[31] A. D. Brailsford, L. K. Mansur. J. Nucl. Mater., 71:110 (1977).

[32] V. I. Dubinko et al., J. Nucl. Mater., 161(1):57 (1989).

[33] L. Shao et al. J. Nucl. Mater., 453(1-3):176 (2014).

[34] M. P. Short, F. A. Garner, L. Shao. "Strong Effect of Injected Interstitials on Suppressing Void Swelling in Ion-Irradiated Iron." RIAR 50 ${ }^{\text {th }}$ Anniversary Symposium, Dimitrovgrad, Russia (April 2014).

[35] B. Singh, T. Leffers, A. Horsewell. Phil. Mag. A, 53(2):233-242 (1986).

[36] F. A. Garner, M. B. Toloczko, B. H. Sencer, J. Nucl. Mater., 276(1-3):123 (2000).

[37] F. A. Garner, "Void swelling and irradiation creep of ferritic-martensitic alloys at very high dpa levels produced by either neutron or self-ion irradiation," Tech Source Science and Engineering Consultants, 2010 ANS Winter Meeting, November 7-11, 2010, Las Vegas, Nevada, USA.

[38] N. I. Budylkin et al. J. Nucl. Mater., 375(3):359 (2008).

[39] F. Hofmann et al. "Lattice swelling and modulus change in a helium implanted tungsten alloy: X-ray micro--diffraction, surface acoustic wave measurements, and multiscale modelling," accessed at http://arxiv.org/ftp/arxiv/papers/1407/1407.6051.pdf on August 26, 2014.

[40] D. Hurley et al. "Mechanical Properties of Nuclear Fuel Surrogates Using Picosecond Laser Ultrasonics.” Idaho National Laboratory Technical Report INL/CON-12-27719 PREPRINT, accessed at http://www.inl.gov/technicalpublications/Documents/5808430.pdf on July 26, 2014.

[41] J. A. Rogers, A. Maznev, K. A. Nelson, "Impulsive stimulated thermal scattering," Charact. Mater., 2:744 (2003).

[42] S. A. Maloy. "Fuel Cycle Research and Development: Core Materials Technologies." Los Alamos National Laboratory Technical Report LA-UR-12-24216, August 2012, accessed at http://permalink.lanl.gov/object/tr?what=info:lanl-repo/lareport/LA-UR-1224216 on August 26, 2014.

[43] R.J. M. Pellenq et al. PNAS, 106:16102 (2009).

[44] E. Masoero et al., PNAS (2014), submitted.

[45] C. A. Muller et al., J. Phys. Chem. 117:403 (2013).

[46] M. J. Abdolhosseini Qomi et al., Nature Comm., 5:4960 (2014).

[47] M. R. Sorensen, A. F. Voter, J. Chem. Phys. 112:9599 (2000).

[48] L. K. Beland et al., Phys. Rev. E 84:046704 (2011).

[49] M. P. Short et al. MRS-B 39(1):71-77 (2014).

[50] Y. Fan, S. Yip, B. Yildiz, J. Phys. Cond. Mat., 26:365402 (2014).

[51] A. Kushima et al., Europ. J. Phys. B 82:271 (2011).

[52] J. W. Bullard et al., Cem. Concr. Res. 41:1208 (2011).

[53] K. Van Vliet et al., MRS Bulletin 37:395 (2012).

[54] Y. Fan et al., Phys. Rev. Lett. 109:135503 (2012). 
[55] M. Fuchs and M. E. Cates, J. Rheol. 53:957 (2009).

[56] G. Tribello, M. Ceriotti, M. Parrinello, PNAS 107:17509 (2010).

[57] A. Barducci, M. Bonomi, M. Parrinello, in Advanced Review, (Wiley, 2011).

[58] J. Antonaglia et al, Phys. Rev. Lett. 112:155501 (2014).

[59] A. El-Azab et al, Curr. Op. Sol. St. Mater. Sci. 18:90 (2014).

[60] F. Mallemace et al., PNAS 107:22457 (2010).

[61] M. Griffiths, R. W. Gilbert, G. J. C. Carpenter, J. Nucl. Mater., 150(1):53 (1987).

[62] J. Li, et al., PloSONE, 6:e17909 (2011).

[63] M. L. Falk, J. S. Langer, Ann. Rev. Condens. Matt. Phys., 2:353 (2011). 